\title{
Tracing the origins of permitted emission lines in RU Lupi down to AU scales ${ }^{\star}$
}

\author{
L. Podio $^{1}$, P. J. V. Garcia ${ }^{2,3}$, F. Bacciotti ${ }^{1}$, S. Antoniucci ${ }^{4}$, B. Nisini ${ }^{4}$, C. Dougados ${ }^{5}$, and M. Takami ${ }^{6}$ \\ 1 INAF - Osservatorio Astrofisico di Arcetri, Largo E. Fermi 5, 50125 Firenze, Italy \\ e-mail: [lindapod; fran]@arcetri.astro.it \\ 2 Centro de Astrofísica da Universidade do Porto, Rua das Estrelas s/n, 4150-762 Porto, Portugal \\ e-mail: pgarcia@astro.up.pt \\ 3 Departamento de Engenharia Física, Faculdade de Engenharia da Universidade do Porto, Portugal \\ 4 INAF - Osservatorio Astronomico di Roma, via di Frascati 33, 00040 Monte Porzio Catone, Italy \\ e-mail: [nisini; antoniucci]@mporzio.astro.it \\ 5 Laboratoire d'Astrophysique de Grenoble, BP 53, 38041 Grenoble Cedex, France \\ e-mail: dougados@obs.ujf-grenoble.fr \\ 6 Institute of Astronomy and Astrophysics, Academia Sinica, PO Box 23-141, Taipei 10617, Taiwan, R.O.C. \\ e-mail: hiro@asiaa.sinica.edu.tw
}

Received 17 September 2007 / Accepted 13 November 2007

\begin{abstract}
Context. Most of the observed emission lines and continuum excess from young accreting low mass stars (Classical T Tauri stars CTTSs) take place in the star-disk or inner disk region. These regions have a complex emission topology that is still largely unknown. Aims. In this paper the magnetospheric accretion and inner wind contributions to the observed permitted $\mathrm{He}$ and $\mathrm{H}$ near infrared (NIR) lines of the bright southern CTTS RU Lupi are investigated for the first time.

Methods. Previous optical observations of RU Lupi have shown a strong $\mathrm{H} \alpha$ profile, due to the emission from a wind in the line wings, and a micro-jet detected in forbidden lines. We extend this analysis to NIR lines through the seeing-limited, high spectral-resolution spectra taken with VLT/ISAAC, and narrow-band imaging and low spectral-resolution spectroscopy acquired with the adaptive optics (AO) aided telescope VLT/NACO. Using spectro-astrometric analysis we investigate the presence of extended emission down to very low spatial scales (a few AU).

Results. The HeI $\lambda 10830$ line presents a P Cygni profile whose absorption feature indicates the presence of an inner stellar wind. Moreover, the spectro-astrometric analysis proves the presence of an extended emission superimposed on the absorption feature and likely coming from the micro-jet detected in the optical. In contrast, the origin of the hydrogen Paschen and Brackett lines is difficult to address. We tried to explain the observed line profiles and flux ratios with both accretion and wind models showing the limits of both approaches. The lack of a spectro-astrometric signal indicates that the HI emission is either compact or symmetric. Our analysis confirms the sensitivity of the HeI line to the faint extended emission regions in the close proximity of the star.
\end{abstract}

Key words. stars: individual: RU Lupi - stars: winds, outflows - accretion, accretion disks - line: formation techniques: high angular resolution

\section{Introduction}

Classical T Tauri stars (CTTSs) are young solar and lower mass stars presenting a rich emission-line spectrum in excess of the continuum from the stellar photosphere. This emission includes hydrogen lines, low-energy forbidden lines, and helium lines, together with a continuum excess - the veiling.

Optical and NIR forbidden emission lines, such as the [O I], [S II], and [Fe II] lines, have large enough emission regions to be resolved, and they are typically associated with outflowing jets (e.g. Bacciotti et al. 2000; Dougados et al. 2000; Pyo et al. 2006). On the other hand, the atomic permitted lines, such as the hydrogen lines, are more difficult to interpret. The energy released by the accretion of matter onto the star can power the permitted line emission that is believed to arise from magnetospheric accretion columns and/or accretion shocks (e.g. Muzerolle et al. 2001;

* Based on observations collected at the European Southern Observatory, Chile (ESO Programme 71.C-0703).
Bouvier et al. 2007). The fact that the emission component of permitted atomic lines is attributed to infalling gas is supported by the presence of inverse P Cygni profiles and by the correlation of their luminosities with the accretion luminosity, derived from the veiling (Muzerolle et al. 1998b,c). On the other hand, the blueshifted absorption features, when present, are an indicator of high-velocity inner winds (e.g. Edwards et al. 1987; Najita et al. 2000). Indeed, several recent spectroscopic studies suggest that the emission of the permitted $\mathrm{HI}$ and $\mathrm{HeI}$ lines can actually include a contribution from both inflowing and outflowing gas (e.g. Beristain et al. 2001; Folha \& Emerson 2001; Takami et al. 2001; Whelan et al. 2004; Edwards et al. 2006). This has motivated hybrid models where the hydrogen emission is due to both the magnetospheric accretion and a wind (Alencar et al. 2005; Kurosawa et al. 2006).

That the collimated, spatially resolved jets, traced at a large distance from the source by optical, near-infrared forbidden lines, are generated in circumstellar regions (less than $10 \mathrm{AU}$ 
from the source) is predicted by theoretical models (Anderson et al. 2003; Ferreira et al. 2006) and observationally proved by the frequent detection of blueshifted absorption features in a number of strong permitted lines ( $\mathrm{H} \alpha, \mathrm{Na}, \mathrm{D}, \mathrm{CaII}, \mathrm{MgII}$, Najita et al. 2000) formed close to the star. Signatures of outflowing gas revealed by absorption features can also be searched in the profile of the HeI $\lambda 10830$ line (Takami et al. 2002; Edwards et al. 2003; Dupree et al. 2005). Edwards et al. (2006) analyzed a sample of $31 \mathrm{~T}$ Tauri stars and show that the HeI line is much more sensitive than the $\mathrm{H} \alpha$ line usually used to trace inner winds in absorption. (In $71 \%$ of the sources was detected an absorption feature in the HeI line against only $10 \%$ of detections in the $\mathrm{H} \alpha$ line.) Beristain et al. (2001) show that the diagnostic power of permitted helium lines in tracing inner winds, lies in their high excitation potential $(20-50 \mathrm{eV})$, which restricts the line formation to a region of either high temperature or close proximity to a source of ionizing radiation.

To investigate the reliability of such scenarios, we observed the circumstellar region of the T Tauri star RU Lupi in the NIR wavelength range. This source is one of the most active and wellknown T Tauri stars (Lamzin et al. 1996; Stempels \& Piskunov 2002; Herczeg et al. 2005) and it is a good target for investigating the inner winds and accretion flows down to very small spatial scales. RU Lupi is located at a distance of only $140 \mathrm{pc}$ (de Zeeuw et al. 1999), allowing us to reach small spatial scales with high angular resolution instrumentation and/or specialized techniques of data analysis. In particular, NACO at the VLT was used to take high angular-resolution images and low spectral-resolution spectra. With VLT/ISAAC seeing-limited and high-spectral resolution spectra were obtained. A spectro-astrometric analysis was applied. Spectro-astrometry is a powerful technique of obtaining positional information on the region originating the emission in the components of a line profile even in seeing-limited conditions. Takami et al. (2001), for example, using spectroastrometry, found that the blueshifted and redshifted wings of the $\mathrm{H} \alpha$ line emitted by RU Lupi show a spatially symmetric offsets with respect to the source, in the same direction as the jet traced, on higher spatial scales, by the forbidden [O I] and [S II] lines. Whelan et al. (2004) detected a similar extended emission in the wings of the $\mathrm{Pa} \beta$ line in three T Tauri sources (DG Tau, V536 Aql, and $\mathrm{LkH} \alpha 321$ ).

In this paper we present the results from such a study and, in particular, a detailed analysis of the origin of the Paschen and Brackett HI lines and the HeI $\lambda 10830$ line. The paper is structured as follows. In Sect. 2 we present the different set of observations and the data reduction process; in Sect. 3 the observed line profiles and fluxes are analyzed; moreover, we present the concepts underlying the spectro-astrometry technique and the obtained position spectra; finally in Sect. 4 we discuss the origin of permitted $\mathrm{H}$ and $\mathrm{He}$ lines by means of a model for wind and accretion and of the results obtained with spectro-astrometry. In the conclusions (Sect. 5) we summarize our findings.

\section{Observations and data reduction}

Observations of RU Lupi were carried out in between April and June 2003 at the ESO Very Large Telescope. The first set of spectra was acquired at the UT4-YEPUN telescope using the infrared camera and spectrometer CONICA, working in cascade to the adaptive optics (AO) system NAOS, and the 86 mas width slit. The AO system allows one to obtain a high spatial resolution of 0.'08-0.' 12 (full width at half maximum - FWHM - of a point source), sampled by a pixel scale of 0 ' $^{\prime} 054$. The second set of data was taken at the UT1-ANTU telescope using ISAAC with the 0.3 slit to obtain a high spectral resolution $(R \sim 10000$; spectral sampling: $\sim 14 \mathrm{~km} \mathrm{~s}^{-1}$; pixel scale: $0{ }^{\prime} 147 /$ pixel), while the spatial resolution is limited by the seeing $(F W H M \sim 0.6-$ 0 ' $^{\prime}$ ). The NACO observations cover all the $J$ and $H$ bands wavelength ranges $(R \sim 400$ and $R \sim 1500$, respectively) while the ISAAC spectra centered at $1.08,1.27$, and $1.588 \mu \mathrm{m}$ cover very short wavelength segments. Both the NACO and the ISAAC spectra were obtained at two slit position angles $\left(217^{\circ}\right.$ and $\left.127^{\circ}\right)$, i.e. parallel and perpendicular to the jet direction as determined by Takami et al. (2001).

In addition, two sets of images of the source in the narrow band filters centered on the HeI $\lambda 10830$ and [FeII] $\lambda 1.25$, $1.64 \mu \mathrm{m}$ lines and around their near continuum (at 1.09, 1.24, and $1.75 \mu \mathrm{m}$ ) were taken with NAOS/CONICA. The information about the spectral observations and the images are summarized in Table 1.

The spectra were reduced using standard IRAF tasks and Eclipse routines from the ESO pipeline. The images were corrected for bad pixels and cosmic rays and for ghosts, then flat-fielded and sky-subtracted. Wavelength calibration was performed using the spectra of the arc lamps for the NACO data and the sky $\mathrm{OH}$ lines for the ISAAC spectra. The systemic motion of the object was subtracted using the radial velocity in the local standard of rest frame, determined by Takami et al. (2001) through the Li $\lambda 6707.815$ absorption line on the stellar atmosphere, $V_{\mathrm{LSR}}=8 \pm 2 \mathrm{~km} \mathrm{~s}^{-1}$. The spectra were corrected for the atmospheric transmission and for the efficiency of the camera, and then flux-calibrated using a telluric spectro-photometric standard. The typical uncertainty for the flux calibration is $\sim 5 \%$. Since the observed telluric stars are of type B, the absorption $\mathrm{HI}$ lines were subtracted before the correction. Unfortunately the $\mathrm{Br} 10, \mathrm{Br} 13$, and $\mathrm{Br} 15$ absorption lines were deeply blended with the atmospheric features in the telluric spectra. In the NACO spectra, because of the low resolution, it was not possible to perform a de-blending and subtract these $\mathrm{HI}$ absorption lines in the spectrum of the telluric. Thus the fluxes and the equivalent widths of the $\mathrm{Br} 10, \mathrm{Br} 13$, and $\mathrm{Br} 15$ lines measured from the NACO spectra are only upper limits. In the ISAAC telluric spectra, on the contrary, the HI lines were de-blended from the atmospheric features and subtracted. Even at the higher spectral resolution of the ISAAC data, however, this process is not very accurate and implies an oversubtraction. For this reason the fluxes, the $F W H M$, and the equivalent widths of the $\mathrm{Br} 10, \mathrm{Br} 13$, and Br15 lines inferred from the ISAAC spectra should be considered as lower limits.

The NACO images were reduced using standard procedures: removal of bad pixel and cosmic rays, dark subtraction, flatfielding, and sky-subtraction. Then the images acquired with the narrow band filters centered on the continuum emission were subtracted from the images filtered on the line emission (HeI and $[\mathrm{Fe} \mathrm{II}]$ ). The subtraction was performed by (i) choosing images with similar PSF, to minimize the effects of a different distribution of the emission in the different filters; (ii) normalizing the images for the source flux estimated through a Gaussian bidimensional fit.

\section{Results}

Analysis of the NACO imaging data showed that no physical companion is detected in the continuum frames in the limit of our spatial resolution $(\sim 14 \mathrm{AU})$. No extended emission structure was detected in continuum subtracted emission line frames at $\mathrm{HeI}$ and $[\mathrm{Fe} \mathrm{II}] \lambda 1.25,1.64 \mu \mathrm{m}$. This constrains the presence of 
Table 1. Instrumental setup during observations.

\begin{tabular}{cccccccc}
\hline \hline Instrument & "'/pix & Slit & Date & $\lambda(\mu \mathrm{m})$ & $R$ & PA & $T_{\text {int }} \times N_{\text {ex }}$ \\
\hline NACO & 0.013 & n.a. & $20 / 25-5-03$ & NB 1.08,1.09 & n.a. & n.a. & $10.8 \mathrm{~s} \times 12$ \\
imaging & & & & NB 1.24, 1.26 & & & $9 \mathrm{~s} \times 12$ \\
& & & & NB 1.64, 1.75 & & & $9 \mathrm{~s} \times 12$ \\
\hline NACO & 0.054 & $0.086 \times 40^{\prime \prime}$ & $16-6-2003$ & J: $0.91-1.37$ & 400 & $217^{\circ}, 127^{\circ}$ & $18 \mathrm{~s} \times 10$ \\
spectroscopy & & & $22-4-2003$ & H: $1.50-1.84$ & 1500 & & \\
\hline ISAAC & 0.147 & $0,3 \times 120^{\prime \prime}$ & $26-6-2003$ & SZ: $1.06-1.1$ & 11500 & $217^{\circ}, 127^{\circ}$ & $300 \mathrm{~s} \times 4$ \\
& & & & J: $1.24-1.3$ & 10500 & $217^{\circ}, 127^{\circ}$ & $300 \mathrm{~s} \times 4$ \\
& & & & H: $1.54-1.62$ & 10000 & $217^{\circ}$ & $250 \mathrm{~s} \times 2$ \\
\hline
\end{tabular}

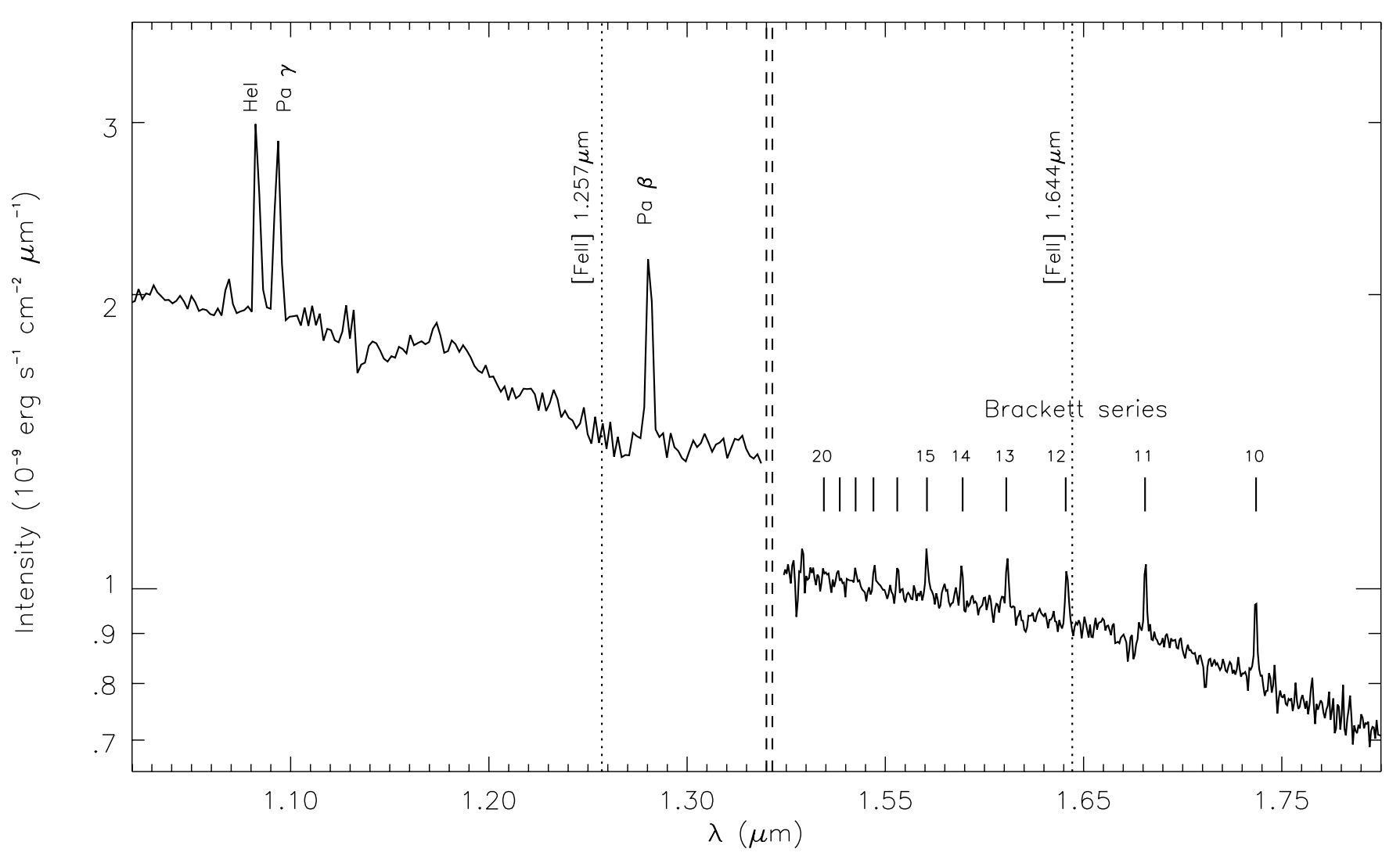

Fig. 1. Low-resolution NACO spectrum of RU Lupi in the $J$ and $H$ bands. The HeI $\lambda 10830$ line and the HI emission lines from the Paschen and the Brackett series are shown. The dotted lines indicate the wavelength position of the [Fe II] lines at 1.25 and $1.64 \mu \mathrm{m}$, which were not detected in our spectra.

wind emission in the HeI and [Fe II] lines over a region smaller than $14 \mathrm{AU}$.

The NACO low-resolution spectra show strong hydrogen recombination lines in emission $(\mathrm{Br} 10$ to $\mathrm{Br} 19$ and $\mathrm{Pa} \beta$, $\mathrm{Pa} \gamma)$ and the HeI $\lambda 10830$ line (see Fig. 1 and Table 2). The ISAAC medium spectral resolution spectra allow us to study the spectral profiles of the $\mathrm{HeI}, \mathrm{Pa} \beta, \mathrm{Pa} \gamma$ and $\mathrm{Br} 13,14,15,16$ lines (see Fig. 2 and Table 3) in more detail, as well as their spatial displacement with respect to the continuum emission through spectro-astrometry (see Sect. 3.3).

No [Fe II] lines are detected at 1.25, 1.32, 1.53, 1.60, and $1.64 \mu \mathrm{m}$ both in the NACO and in the ISAAC spectra. The lack of [Fe II] emission is not expected in this source. In fact, studies of $\mathrm{HH}$ jets in the optical and in the NIR ranges (Podio et al. 2006) showed that optical jets also emit in the NIR [Fe II] lines, unless the density in the jet beam is very low $\left(n_{\mathrm{e}}<10^{3} \mathrm{~cm}^{-3}\right)$. In RU Lupi, indeed, strong and spatially extended emission in the optical forbidden lines ([S II], [O I], and [N II]) was observed by Takami et al. (2001); on the other hand, electron densities higher than $10^{4}-10^{5} \mathrm{~cm}^{-3}$ were estimated by the same authors based on the [S II] lines' equivalent-width ratio. Therefore, iron lines should be present in the spectra. Possible reasons for not detecting $\mathrm{Fe}$ emission could be that a short integration time was used, or that a strong depletion of iron caused a fainter emission than the predicted one. In fact, an increase depletion near to the source has been observed in other jet sources as, e.g. HH 1 (Nisini et al. 2005).

\subsection{HI emission lines}

The availability of complementary data sets (see Table 1) allows us to investigate the origin of HI lines through both the measured line fluxes and the line profiles. The characteristics and the parameters of the lines profiles can be retrieved from the ISAAC spectra thanks to the high spectral resolution. The center $\left(V_{\text {peak }}\right)$, the $F W H M(\Delta V)$, the equivalent width $(E W)$, and the dereddened flux (F) of the HI lines that are in the spectral range covered by the ISAAC spectra $(\mathrm{Pa} \beta, \mathrm{Pa} \gamma$ and $\mathrm{Br} 13,14,15$, and 16) are 
Table 2. Equivalent widths $(E W)$ and dereddened line fluxes $(F)$ from NACO spectroscopy.

\begin{tabular}{cccc}
\hline \hline $\begin{array}{c}\lambda \\
\mu \mathrm{m}\end{array}$ & Line & $\begin{array}{c}E W \\
\mathrm{~km} \mathrm{~s}^{-1}\end{array}$ & $\begin{array}{c}F \\
10^{-13} \mathrm{erg} \mathrm{s}^{-1} \mathrm{~cm}^{-2}\end{array}$ \\
\hline 1.0830 & $\mathrm{HeI}$ & 452 & $40 \pm 5$ \\
1.0941 & $\mathrm{~Pa} \gamma$ & 457 & $40 \pm 5$ \\
1.2822 & $\mathrm{~Pa} \beta$ & 434 & $32 \pm 3$ \\
1.5265 & $\mathrm{Br} 19$ & 9 & $0.5 \pm 0.2$ \\
1.5346 & $\mathrm{Br} 18$ & 14 & $0.8 \pm 0.2$ \\
1.5443 & $\mathrm{Br} 17$ & 20 & $1.1 \pm 0.2$ \\
1.5561 & $\mathrm{Br} 16$ & 19 & $1.1 \pm 0.1$ \\
1.5705 & $\mathrm{Br} 15$ & 46 & $2.7 \pm 0.3^{*}$ \\
1.5885 & $\mathrm{Br} 14$ & 31 & $1.8 \pm 0.2$ \\
1.6114 & $\mathrm{Br} 13$ & 55 & $3.1 \pm 0.3^{*}$ \\
1.6412 & $\mathrm{Br} 12$ & 48 & $2.7 \pm 0.3$ \\
1.6811 & $\mathrm{Br} 11$ & 63 & $3.5 \pm 0.3$ \\
1.7367 & $\mathrm{Br} 10$ & 78 & $3.9 \pm 0.3^{*}$ \\
\hline
\end{tabular}

* The equivalent widths $(E W)$ and the fluxes $(F)$ of the $\operatorname{Br} 10, \operatorname{Br} 13$, and $\mathrm{Br} 15$ line are only upper limits to the real flux, due to the presence of $\mathrm{HI}$ lines blended with atmospheric features in the spectrum of the telluric standard (see Sect. 2).

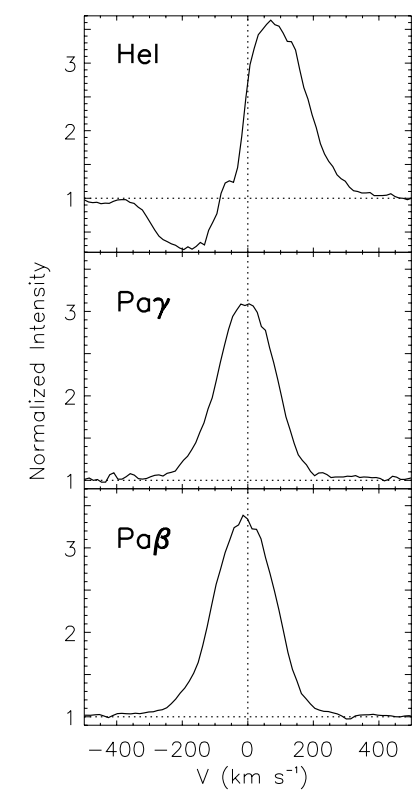

Fig. 2. ISAAC spectra of the $\mathrm{HeI} \lambda 10830, \mathrm{~Pa} \gamma$ and $\mathrm{Pa} \beta$ lines. The lines are normalized to the continuum intensity.

summarized in Table 3. The classification proposed by Reipurth et al. (1996) for the $\mathrm{H} \alpha$ line profiles has already been used for NIR hydrogen lines by Folha \& Emerson (2001) and Whelan et al. (2004). According to this classification scheme, the detected HI lines are type I profiles; i.e. they are nearly symmetric showing no evidence of absorption features, but they have large FWHM and extended wings. All the Paschen and Brackett lines detected are, in fact, broad $\left(\Delta V \sim 200 \mathrm{~km} \mathrm{~s}^{-1}\right.$, except for the $\mathrm{Br} 13$ and the $\mathrm{Br} 15$ lines for which we get only a lower limit to the $F W H M)$ and slightly blueshifted $\left(-5<V_{\text {peak }}<-30\right)$. In particular, the intensity profiles normalized to the continuum emission of the $\mathrm{Pa} \gamma$ and $\mathrm{Pa} \beta$ lines are shown in Fig. 2. Both the $\mathrm{Pa} \gamma$ and $\mathrm{Pa} \beta$ lines have a broad nearly symmetric profile peaking at 3-3.5 times the intensity of the continuum. The profile is centered at -5 and $-11 \mathrm{~km} \mathrm{~s}^{-1}$, respectively, and extends at least from $-300 \mathrm{~km} \mathrm{~s}^{-1}$ to $+200 \mathrm{~km} \mathrm{~s}^{-1}$.
Table 3. Line properties from ISAAC spectroscopy.

\begin{tabular}{cccccc}
\hline \hline $\begin{array}{c}\lambda \\
\mu \mathrm{m}\end{array}$ & Line & $\begin{array}{c}V_{\text {peak }} \\
\mathrm{km} \mathrm{s}^{-1}\end{array}$ & $\begin{array}{c}\Delta V \\
\mathrm{~km} \mathrm{~s}^{-1}\end{array}$ & $\begin{array}{c}E W \\
\mathrm{~km} \mathrm{~s}^{-1}\end{array}$ & $\begin{array}{c}F^{a} \\
10^{-13} \mathrm{erg} \mathrm{s}^{-1} \mathrm{~cm}^{-2}\end{array}$ \\
\hline${ }^{b} 1.0830$ & HeI ab & -200 & - & 133 & - \\
${ }^{c} 1.0830$ & $\mathrm{HeI} \mathrm{em}$ & 70 & - & 581 & $47 \pm 6$ \\
1.0941 & $\mathrm{~Pa} \gamma$ & -5 & 206 & 475 & $40 \pm 5$ \\
1.2822 & $\mathrm{~Pa} \beta$ & -11 & 214 & 562 & $38 \pm 3$ \\
1.5561 & $\mathrm{Br} 16$ & -31 & 179 & 23 & $1.2 \pm 0.2$ \\
${ }^{*} 1.5705$ & $\mathrm{Br} 15$ & -16 & 128 & 20 & $1.0 \pm 0.2$ \\
1.5885 & $\mathrm{Br} 14$ & -22 & 185 & 43 & $2.2 \pm 0.2$ \\
${ }^{*} 1.6114$ & $\mathrm{Br} 13$ & -19 & 159 & 44 & $2.7 \pm 0.3$ \\
\hline
\end{tabular}

${ }^{a}$ The fluxes $(F)$ are corrected for dust extinction (see Sect. 3.1).

$b, c$ Parameters relative to the absorption (ab) and emission (em) part of the HeI line profile (see Fig. 2).

${ }^{*}$ Due to the presence of HI lines blended with atmospheric features in the spectrum of the telluric standard, the parameters inferred for the $\mathrm{Br} 13$ and $\mathrm{Br} 15$ lines are only rough estimates (see Sect. 2). In particular the width, $\Delta V$, and the flux, $F$, are lower limits.

The ratios between the lines of the Paschen and Brackett series can be used to retrieve further information about the origin of the HI lines. The NACO spectra have spectral resolution too low to derive the properties of the line profiles $\left(V_{\text {peak }}, \Delta V\right)$. They can be used, however, to measure the equivalent widths and the fluxes of the Paschen and Brackett lines (see Table 2). With respect to the ISAAC data, these spectra have the advantage of covering all the Brackett lines from the $\mathrm{Br} 10$ up to the $\mathrm{Br} 19$ in the same spectral segment, allowing us to measure and compare the fluxes of all the lines without introducing calibration problems.

First, we corrected the observed fluxes for the reddening. We used the average value of the visual extinction estimated by Giovannelli et al. (1995) in the circumstellar region of RU Lupi $\left(A_{V}=0.7 \pm 0.3\right)$ and the extinction law derived by Rieke \& Lebofsky (1985). The dereddened line fluxes of the Paschen and the Brackett lines are summarized in Table 2 for the NACO data and in Table 3 for the ISAAC ones. The error affecting the line fluxes and their ratios is computed by considering the noise over the continuum and the uncertainty on the $A_{V}$ value. Then we computed the Brackett decrement, i.e. the ratios between the intensity of the Brackett lines coming from the upper level $n$ and the first line of the series. The first Brackett line in the spectral range covered by our spectra is the $\mathrm{Br} 10$, but this line is not corrected well for the atmospheric transmission (see Sect. 2). Thus we used the ratios with the Brackett 11 line. The Brackett ratios, $\operatorname{Br} n / \operatorname{Br} 11$, as a function of the upper level $n$, are plotted in Fig. 3. (The ratios $\mathrm{Br} 15 / \mathrm{Br} 11$ and $\mathrm{Br} 13 / \mathrm{Br} 11$ are not taken into consideration since for the Br13 and Br15 lines we have only an upper limit of the flux, see Sect. 2.) In the figure we overplotted the theoretical values of the Brackett ratios calculated for two different cases. The plot shows that the ratios between the Brackett lines are well-fitted by case B recombination, i.e. by optically thin emission. As we discuss in Sect. 4.1, however, case B cannot reproduce the observed fluxes for typical values of the emitting volume.

To avoid systematic errors that can affect the lines of the same series and to constrain the emission conditions even more tightly, we also considered the ratio between the observed Paschen lines, $\mathrm{Pa} \beta / \mathrm{Pa} \gamma$. The measured $\mathrm{Pa} \beta / \mathrm{Pa} \gamma$ ratio retrieved from the NACO spectra is $\sim 0.8 \pm 0.1$, and the one retrieved from the ISAAC ones is $\sim 1 \pm 0.1$. Such values cannot be produced by the case $B$ recombination. In fact, for any choice of the electron density and the temperature between $10^{2}$ and $10^{10} \mathrm{~cm}^{-3}$ and 


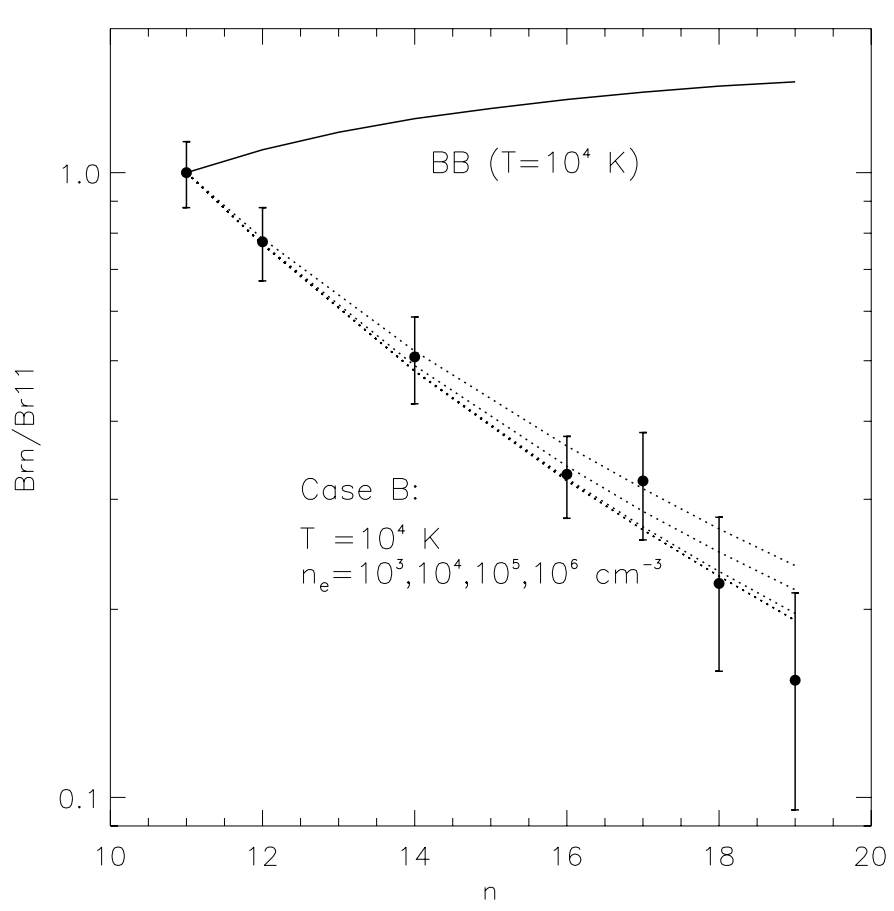

Fig. 3. Ratios of the Brackett lines with respect to $\mathrm{Br} 11$ versus the upper quantum number $n$. The solid line indicates the theoretical value of the ratios expected in case of optically thick emission at $T=10000 \mathrm{~K}$ (black-body emission). The four dotted lines are the ratios expected from case B recombination (Hummer \& Storey 1987) assuming $T=$ $10000 \mathrm{~K}$ and $n_{\mathrm{e}}=10^{3}, 10^{4}, 10^{5}, 10^{6} \mathrm{~cm}^{-3}$ going from the lower to the upper line. Note that for the considered values of the electron density, variations in the temperature between $10^{3}$ and $10^{5} \mathrm{~K}$ leave the curves almost identical.

$5 \times 10^{2}$ and $3 \times 10^{4} \mathrm{~K}$, respectively, case $\mathrm{B}$ gives $\mathrm{Pa} \beta / \mathrm{Pa} \gamma>1.4$. On the other hand, in the case of optically thick emission (blackbody emission), the $\mathrm{Pa} \beta / \mathrm{Pa} \gamma$ ratio is $\sim 0.6$ at $T=10^{4} \mathrm{~K}$ and $\sim 0.7$ at $T=5 \times 10^{3} \mathrm{~K}$. This implies that the Paschen lines are not reproduced by one of these limiting cases and that their ratio strongly depends on their relative optical depth.

\subsection{The Hel $\lambda 10830$ line}

The HeI line profile obtained from medium-resolution ISAAC spectra (Fig. 2) shows a typical P-Cygni profile with the emission part peaking at $+70 \mathrm{~km} \mathrm{~s}^{-1}$ and extending up to at least $+430 \mathrm{~km} \mathrm{~s}^{-1}$, and a broad blueshifted absorption penetrating up to $80 \%$ of the continuum at $V \sim-200 \mathrm{~km} \mathrm{~s}^{-1}$ and ranging from $-360 \mathrm{~km} \mathrm{~s}^{-1}$ to $-90 \mathrm{~km} \mathrm{~s}^{-1}$ (see Table 3).

The absorption feature clearly indicates the presence of an inner wind (Edwards et al. 2003). The efficiency of the HeI $\lambda 10830$ transition in tracing outflowing gas is due to the "metastability" of its lower level $\left(2 \mathrm{~s}^{3} \mathrm{~S}\right)$. Even if energetically far above the singlet ground state $(21 \mathrm{eV})$, this level is radiatively isolated from it, and collisionally de-excitation is very low. This means that, once it is populated, via photoionization followed by recombination and cascade and/or via collisional excitation from the ground state, this level becomes easily over-populated. The absorption feature is generated mainly via resonant scattering since the $\lambda 10830$ transition is the only permitted radiative transition from its upper state to a lower one and the electron density is unlikely to be high enough to cause collisional excitation or de-excitation.
Edwards et al. $(2003,2006)$ demonstrate that the shape and width of the HeI $\lambda 10830$ line can constrain the wind launch region. They show that a broad and deep blue absorption, like the one detected in our spectra, require formation in a wind emerging radially from the star rather than from the disk. In fact, the broad range of velocities covered by the absorption feature can be explained by radially emerging wind streamlines absorbing the $1 \mu \mathrm{m}$ continuum from the stellar surface, thus tracing the full acceleration region of the inner wind. On the contrary, a disk- or $x$-wind is confined to nearly parallel streamlines emerging at an angle to the disk surface and thus the continuum photons from the star will intercept a narrower range of velocities giving rise to narrow absorption feature such as the ones shown in Edwards et al. (2006). In conclusion, the absorption feature in our spectra appears to indicate an inner wind radially emerging from the star. Deeper insight into the geometry and the presence of different wind contributions to the HeI $\lambda 10830$ line can be retrieved from the spectro-astrometric analysis presented in Sect. 3.3 and discussed in Sect. 4.2.

The emission part of the line, instead, is produced by the accreting gas columns and, partially, by the resonant scattering process. The contribution from the latter depends on the electron density and in our case should be much lower than the loss of flux in the absorption part of the line. In fact, the detection of the absorption feature indicates that the efficiency of collisional de-excitation is much lower with respect to the radiative decay.

\subsection{Spectro-astrometry}

Besides the standard spectroscopic analysis, we performed a spectro-astrometric analysis of the data. Following this procedure, the displacement of the emission centroid of the lines with respect to the source continuum is determined (e.g., Bailey 1998). This means that one can detect extended emission in a line beyond the seeing limit, down to (sub-)AU scales. On the other hand, the technique is only sensitive to asymmetric emission and, since it measures a barycenter, it gives only a lower limit to the size of the emission region. The technique consists in accurately measuring the trace of each A-B spectrum by Gaussian-fitting the spectrum spatial profile at each wavelength. In this way one recovers the wavelength-dependent centroid (i.e. the so-called "position spectrum") and Gaussian $F W H M$ of the spectra. The residual FWHM, obtained by subtracting the $F W H M$ of the continuum emission, can be sensitive to differential size variations in the emission region. Examples of the application of spectro-astrometry can be found in Takami et al. (2001) and Whelan et al. (2005).

The essential requirements for this kind of analysis are a good spectral and spatial sampling and a high signal-to-noise ratio $(S N R)$. Thus we only applied spectro-astrometry to the ISAAC spectra, in which the lines are very well-resolved (SNR $100)$ and well-sampled spectrally $\left(\Delta V \sim 200 \mathrm{~km} \mathrm{~s}^{-1}\right.$, spectral sampling $\sim 14 \mathrm{~km} \mathrm{~s}^{-1}$ ), while the spatial sampling is moderately

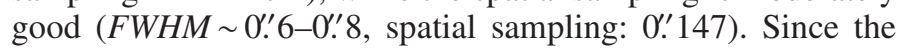
trace of the star is tilted in the ISAAC spectrograph, the centroid is corrected for this distortion with a polynomial fit. The typical spectro-astrometric error is given by the accuracy in measuring the centroid of the spatial profile through the Gaussian fit and is equal to $\sigma \sim 0.5 \mathrm{FWHM} / \sqrt{N_{\mathrm{ph}}}$ (Takami et al. 2003). For the considered lines $\left(N_{\mathrm{ph}}>10^{5}\right)$, this accuracy turns out to be $\sim 1$ mas. In the case of the He line, however, the signal is fainter in the absorption part $\left(N_{\mathrm{ph}} \sim 10^{4}-10^{5}\right)$. Therefore, we adopted $\sigma \sim 3$ mas, which is the average value of the error across the line profile. 
Pa $1.09 \mu \mathrm{m}$

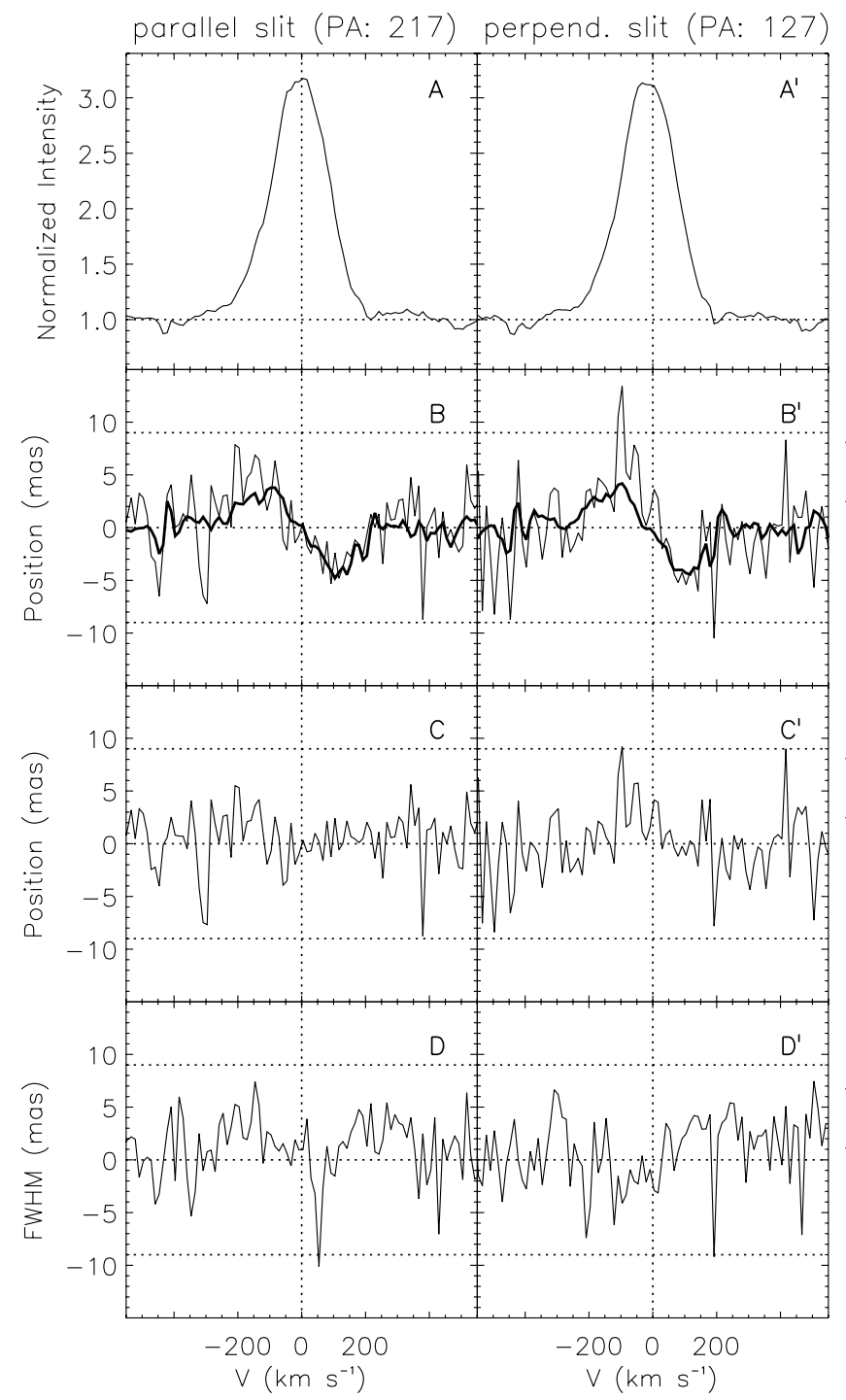

Hel $1.08 \mu \mathrm{m}$

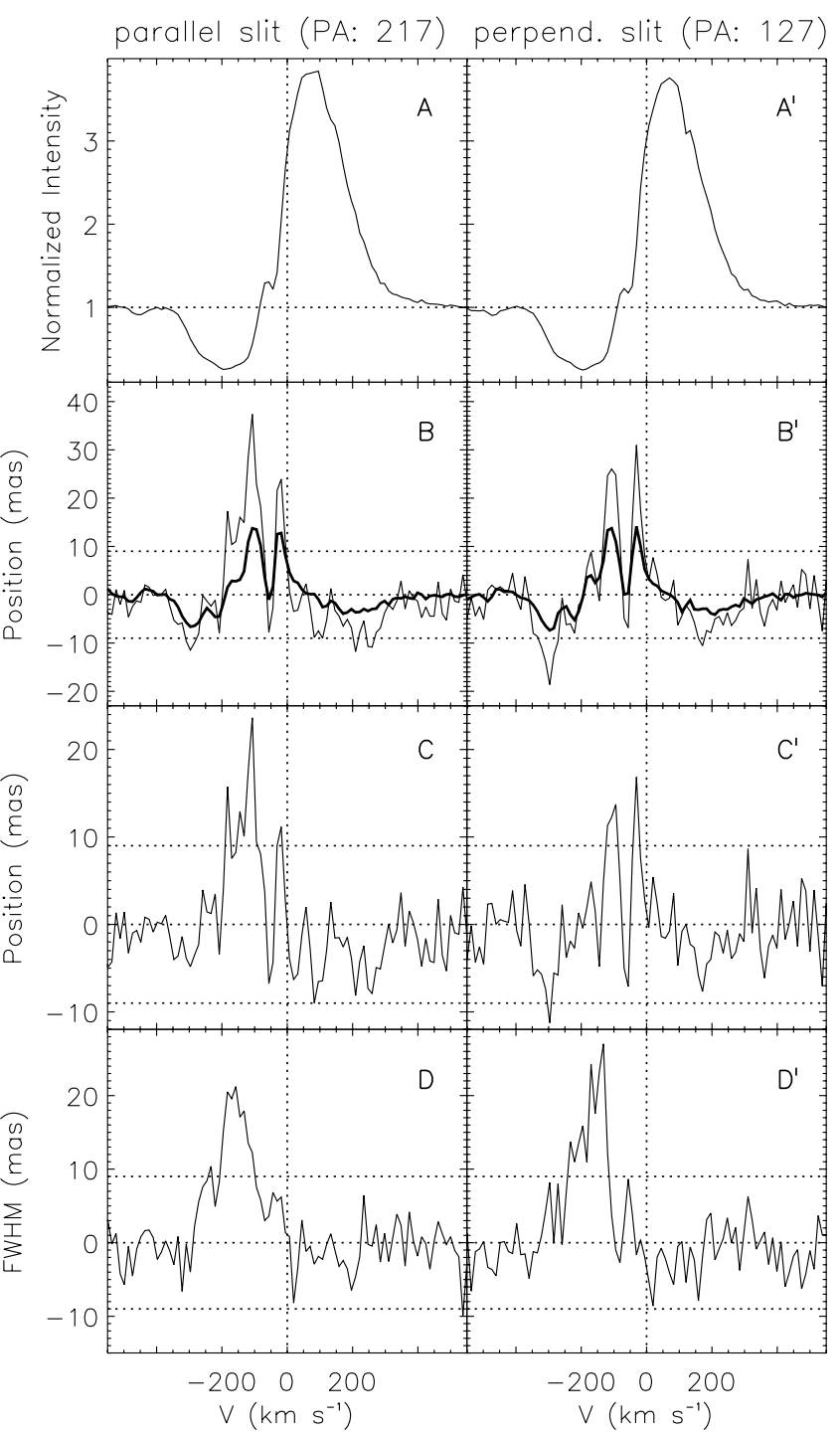

Fig. 4. ISAAC spectroscopy of RU Lupi Pay (left) and HeI (right), with the slit parallel to the jet and perpendicular. The line profiles normalized to the continuum emission are presented in the upper panels - $\mathrm{A}$ and $\mathrm{A}^{\prime}$. The spectro-astrometric signal is presented in the second panels - $\mathrm{B}$ and $\mathrm{B}^{\prime}$. The overplotted solid line is the spectro-astrometric signal due to a distorted PSF caused by tracking error or unstable active optics. The signal is simulated following the procedure of Brannigan et al. (2006) (see text). The residual signal obtained by subtracting the simulated signal to the detected one is shown in the third panels $-\mathrm{C}$ and $\mathrm{C}^{\prime}$. Finally, the residual FWHM of the Gaussian fit is presented in the lower panels $-\mathrm{D}$ and $\mathrm{D}^{\prime}$. The vertical dotted lines show the zero velocity of the line. The horizontal dotted lines indicate the continuum emission level and the $\pm 3 \sigma$ threshold due to the signal-to-noise.

Moreover, since the spatial sampling for the ISAAC data is not exceptional, we used a conservative value for the threshold of $3 \sigma$ (see Fig. 4).

We checked the position spectra for the presence of artifacts following the procedure of Brannigan et al. (2006). These authors show that the distortion of the PSF caused by tracking errors of the telescope or unstable active optics during an exposure can induce artificial signals. These instrumental biases can be simulated in shape and magnitude by calculating the spectroastrometric signal obtained from a modeled distorted PSF. We used the same procedure than in Brannigan et al. (2006) to simulate the biases that can be produced with our instrumental setting (cf. Table 1). The shape of the artifacts produced by a distorted PSF depends on the slope of the line profile. In particular, the peaks of the simulated biases appear at velocities where the gradient of the line profile is the steepest. This explains the double peak of the simulated spectro-astrometric signal in the blueshifted part of the HeI line (see panel B and B' of Fig. 4). The magnitude of such biases, instead, depends on the amplitude of the distortion of the PSF with respect to the slit axis, i.e. on the parameters $\Delta x_{1}, \Delta x_{2}, \Delta y_{1}, \Delta y_{2}$ in Eq. (4) of Brannigan et al. (2006). The maximum biases are obtained when the distortion of the PSF in the slit is at its maximum, i.e. when we put in Eq. (4) of Brannigan et al. (2006) $\Delta x_{1}=\Delta x_{2}=0$ '. 15, $\Delta y_{1}=\Delta y_{2}=0.15$, so that the total slit illumination displacement $(\Delta y)$ is equal to the slit width, 0.3 . The resulting spurious signal was then subtracted from the position spectra.

The position spectra obtained for the $\operatorname{Pa} \beta$ and Brackett lines do not show any shift in the centroid with respect to the continuum emission. On the other hand, in the Pay and the HeI line 
we found a spectro-astrometric signal (see Fig. 4). The position spectrum of these lines, in fact, indicate a shift in the centroid of the emission with respect to the continuum emission both in the parallel and in the perpendicular slit (panels B and $\mathrm{B}^{\prime}$ ). However, our analysis shows that there is no real signal in the Pa $\gamma$ line. In fact, there is no residual in the position spectra of this line after the subtraction of the biases, as well as in the spatial FWHM across the profile (see panels $\mathrm{C}, \mathrm{C}^{\prime}$ and $\mathrm{D}, \mathrm{D}^{\prime}$ ).

In the HeI line, on the contrary, the position spectra cleaned by artifacts (panels $\mathrm{C}$ and $\mathrm{C}^{\prime}$ ) show a shift over the signal-to-noise limit ( 9 mas, corresponding to $\sim 1.3 \mathrm{AU}$ ) in the blueshifted part of the line. The residual FWHM across the profile presents a significant signal in the same velocity range as where the spectro-astrometric signal is detected $\left(-50 \mathrm{~km} \mathrm{~s}^{-1}<V<-200 \mathrm{~km} \mathrm{~s}^{-1}\right)$, reaching differential sizes of 0 . $^{\prime} 025$ (panels D and $\mathrm{D}^{\prime}$ ). A broader FWHM is expected in the region where the absorption feature is detected, because the continuum emission is much fainter. It is, however, interesting to notice that (i) the spatial profile can be identified very well over the background noise across the entire HeI spectral profile (the signal-to-noise ratio is $S / N \sim 100$ in the spectral region covered by the absorption feature and $\sim 10$ times higher in the emission part of the line), (ii) the broadening of the line is not symmetric with respect to the peak of the absorption feature $\left(V_{\text {peak }} \sim-200 \mathrm{~km} \mathrm{~s}^{-1}\right)$. Instead, a constant or increasing $F W H M$ in the parallel and perpendicular slit, respectively, is measured in the region where the spectro-astrometric signal is detected $\left(-200 \mathrm{~km} \mathrm{~s}^{-1}<V<-100 \mathrm{~km} \mathrm{~s}^{-1}\right)$. This suggests that multiple spatial components are contributing to the line profile in this region of the "P Cygni" absorption feature. The interpretation of the detected spectro-astrometric signal is discussed in Sect. 4.2.

\section{Discussion}

As illustrated in the introduction, the excitation mechanism of the HI lines is an actively debated topic. Up to a few years ago, both the observations and the models focused on the Balmer lines and, in particular, on the $\mathrm{H} \alpha$ line. The large amount of optical observations evidenced the limits of both the accretion and the wind models in reproducing the Balmer lines and motivated the development of hybrid models with more complex geometries (e.g., Kurosawa et al. 2006). On the other hand, the capacity of these models to reproduce the profiles and fluxes of the HI infrared lines was poorly tested. Interestingly, the observations of Paschen and Brackett lines, such as the ones presented in Folha \& Emerson (2001), Nisini et al. (2004), Whelan et al. (2004), and Edwards et al. (2006), have shown that the magnetospheric accretion models cannot fully reproduce the characteristics of the observed profiles, again in favor of an hybrid wind/accretion model.

As we have shown in the previous section, the Paschen and the Brackett lines in our spectra present blueshifted broad symmetric profiles $\left(V_{\text {peak }} \sim-17 \mathrm{~km} \mathrm{~s}^{-1}\right.$ and $\left.\Delta V \sim 200 \mathrm{~km} \mathrm{~s}^{-1}\right)$. These are difficult to reproduce if one separately adopts a wind model or, alternatively, a magnetospheric accretion model. Wind models generally predict redshifted peaks and strongly asymmetric profiles with P-Cygni absorption features, such as the one detected in the HeI line (Calvet et al. 1992). Hartmann et al. (1990) were able to reproduce more symmetric $\operatorname{Br} \gamma$ and $\operatorname{Br} \alpha$ profiles, without the P-Cygni absorption feature with a model of a magnetically-driven wind, but their peaks are still redshifted. Edwards et al. (1987) showed that an optically thick circumstellar disk may cause the occultation of the redshifted part of the profile thus explaining blueshifted peaks. In all cases, asymmetric profiles are expected.

On the other hand, magnetospheric accretion models (Muzerolle et al. 1998a) predict blueshifted peaks, but asymmetric and narrow profiles $\left(\Delta V \sim 100 \mathrm{~km} \mathrm{~s}^{-1}\right)$ often showing inverse P-Cygni absorption. Muzerolle et al. (2001) retrieve more symmetric and broad line profiles from their accretion model, taking into account line damping due to different broadening mechanisms, but this effect is really strong only for $\mathrm{H} \alpha$ and Balmer lines, while it is negligible for Paschen and Brackett lines.

We now examine how the above scenarios fit in the case of our RU Lupi spectra.

\subsection{Accretion vs. wind}

As illustrated in Sect. 3.1 the case B recombination reproduces the Brackett decrement, but not the $\mathrm{Pa} \beta / \mathrm{Pa} \gamma$ ratio, whose value implies that these two lines are optically thick. Moreover, case B recombination assumes (i) a constant density, which is not applicable to the circumstellar region of T Tauri stars; and (ii) optically thin lines. To reproduce the observed fluxes, however, the latter hypothesis would imply an emitting volume corresponding to a spherical envelope extending up to $40 \mathrm{AU}$ from the source, which is unrealistic according to the models of circumstellar accretion/ejection structure (e.g., Nisini et al. 2004). To investigate the origin of the HI lines one has to assume a variable density profile, and the optical depth and the flux of the Paschen and Brackett lines will be retrieved when integrating along the line of sight.

Nisini et al. (2004) analyzed the Brackett lines in the Class I source HH 100 IR, finding very similar line profiles to the ones of our Paschen and Brackett lines. Interestingly, they found that the Brackett decrement in HH 100 IR is reproduced neither by the Case B recombination curves (i.e. optically thin emission) nor by the blackbody emission (i.e. completely thick emission). The authors suggest that these lines are emitted in an expanding envelope of ionized hydrogen, and thus different lines are tracing different layers of the envelope according to their optical depth. Following this idea they were able to reproduce the ratios between the optically thick Brackett lines through a simple model of spherical wind.

We used the same approach to reproduce the $\mathrm{Br} n / \mathrm{Br} 11$ ratios (Fig. 3), the $\mathrm{Pa} \beta / \mathrm{Pa} \gamma$ ratio, and the observed line fluxes both in the accretion and in the wind hypothesis adopting a toy model consisting in a spherical envelope of ionized hydrogen, extending from an inner radius $r_{\mathrm{i}}$ to an outer radius $r_{\text {out }}$, where LTE conditions are assumed. If the envelope is expanding (wind hypothesis) or infalling (accretion hypothesis), the radiation transfer can be treated in the Sobolev approximation following the formalism described in Castor (1970) and Nisini et al. (1995). The gas can be only partially ionized, and, therefore, the envelope total mass loss or accretion rate is $\dot{M}_{\text {wind } / \text { accr }}=\dot{M}_{\text {ion }} / x_{\mathrm{e}}$, where $x_{\mathrm{e}}$ is the ionization fraction. The electron density in the envelope is given by the continuity equation, $n_{\mathrm{e}}=\dot{M}_{\text {wind } / \text { accr }} x_{\mathrm{e}} / 4 \pi m_{\mathrm{H}} r^{2} V(r)$.

Using this model both the wind and the accretion hypothesis can be tested by just changing the velocity law $V(r)$. In fact, this will imply a different density profile and thus a difference in the relative optical depths of the lines in the two cases.

In the wind hypothesis the envelope is expanding and the gas velocity follows a general law of the type $V(r)=V_{0}+\left(V_{\max }-\right.$ $\left.V_{0}\right)\left(1-\left(r_{\mathrm{i}} / r\right)^{\alpha}\right)$; i.e. the gas is accelerated from an initial velocity at the base of the wind, $V_{0}$, at the velocity $V_{\max }$ at a distance that depends on the parameter $\alpha$ (Nisini et al. 2004), where $V_{\max }$ is the maximum velocity of the expanding gas. The $F W H M$ of the 
observed lines is determined by the velocity of the gas particles, thus we retrieve $V_{\max } \sim 200 \mathrm{~km} \mathrm{~s}^{-1}$ from the observed profiles.

The simulated emission is tuned by a proper choice of the input parameters. For typical values of the size of the envelope $\left(r_{\mathrm{i}}=1 R_{*}, r_{\text {out }}=3-10 R_{*}\right)$ and of the electron temperature $\left(T=10^{4} \mathrm{~K}\right)$, and taking $\alpha=4$ and $V_{0}=30 \mathrm{~km} \mathrm{~s}^{-1}$ as in Nisini et al. (2004), we are able to reproduce the Brn/Br11 ratios and the absolute fluxes of the Paschen and almost all the Brackett lines (within $\pm 4 \sigma$ ) for $\dot{M}_{\text {ion }} \sim 2 \times 10^{-8} M_{\odot} \mathrm{yr}^{-1}$. On the other hand, in this way we are not able to reproduce the observed $\mathrm{Pa} \beta / \mathrm{Pa} \gamma$ ratio $(\mathrm{Pa} \beta / \mathrm{Pa} \gamma \sim 1.3-1.4)$. Considering a slower acceleration of the wind $(\alpha=1-3)$ and/or a lower temperature ( $T$ down to $5 \times 10^{3} \mathrm{~K}$ ), the $\mathrm{Pa} \beta / \mathrm{Pa} \gamma$ ratio decreases to $\sim 1-1.3$ and the absolute fluxes agree with the observed ones, but the Brackett ratios are not reproduced because the lines optical depths are too large. Taking lower values of the mass loss rate $\left(\dot{M}_{\text {ion }}<10^{-8} M_{\odot} \mathrm{yr}^{-1}\right)$, which is the fundamental parameter regulating the emission in the HI lines, we obtain a good fit of the Brackett decrement for $r_{\text {out }}$ within 3-10 $R_{*}$ and values of the exponent in the acceleration law of the wind between 1 and 4 . The absolute fluxes, however, are too low in this case (3-4 times lower than the observed ones) and the $\mathrm{Pa} \beta / \mathrm{Pa} \gamma$ ratio is $>1.4$. To obtain the required $\mathrm{Pa} \beta / \mathrm{Pa} \gamma$, one should assume a value of $\dot{M}_{\text {ion }}$ higher than $2 \times 10^{-8} M_{\odot} \mathrm{yr}^{-1}$. For such a massive wind, however, the Brackett decrement and line fluxes would no longer be fitted by the model because their optical depth would be too great.

Since we could not find a choice of the parameters for the wind model, which reproduces the Brackett decrement and the observed $\mathrm{Pa} \beta / \mathrm{Pa} \gamma$ ratio at the same time, we tried to use the model to simulate the emission from a spherical accreting envelope by reversing the sign of the gas speed. A spherical model is not the best approximation of the accretion process, which, according to the most recent models, proceeds along the magnetic field lines, in an axisymmetric non-spherical geometry (Hartmann et al. 1994; Muzerolle et al. 1998a). Nevertheless, we have adopted the spherical model as a first approximation, in order to check if the observed ratios are reproduced better by accretion than by an outflow.

If we assume that the HI lines are mainly excited in the accretion columns, we can derive an estimate of the mass accretion rate from the luminosity of the $\mathrm{Pa} \beta$ line. To this aim, we use the empirical relation between $\mathrm{L}(\mathrm{Pa} \beta)$ and the accretion luminosity derived by Muzerolle et al. (1998c) and Natta et al. (2002):

$\log L_{\mathrm{acc}} / L_{\odot}=1.36 \log L(\mathrm{~Pa} \beta) / L_{\odot}+4$.

The mass accretion rate is then computed from $L_{\text {acc }}$ as $\dot{M}_{\text {acc }}=$ $L_{\mathrm{acc}} R_{*} /\left(G M_{*}\right)$. Using for RU Lupi $M_{*}=0.8 M_{\odot}$ and $R_{*}=1.6 R_{\odot}$ (Lamzin et al. 1996; Herczeg et al. 2005), we obtain $\dot{M}_{\text {acc }}=$ $2 \times 10^{-7} M_{\odot} \mathrm{yr}^{-1}$. This value agrees with previous estimates of Lamzin et al. (1996), which tuned the value of $\dot{M}_{\text {acc }}$ to reproduce the observed continuum energy distribution through their model (see, however, Herczeg et al. 2005, for an alternative derivation of $\dot{M}_{\text {acc }}$ using the accretion luminosity estimated from the UV excess).

In the case of an accreting envelope, the typical velocity law is $V(r)=V_{\max } r^{-0.5}$. We fixed the size of the envelope and the temperature $\left(r_{\mathrm{i}}=R_{*}, r_{\text {out }}=3 R_{*}, T=10^{4} \mathrm{~K}\right)$ and examined the results obtained by varying the ionization fraction $x_{\mathrm{e}}$, and thus the electron density of the accreting columns for $\dot{M}_{\text {acc }}=2 \times 10^{-7} M_{\odot} \mathrm{yr}^{-1}$. If the gas is fully ionized $\left(x_{\mathrm{e}}=1\right)$, we obtain $\mathrm{Pa} \beta / \mathrm{Pa} \gamma \sim 0.7$, but the Brackett decrement is not reproduced, because the optical depth of these lines is too large, and the absolute fluxes of all the lines are higher than the observed one by one order of magnitude. Indeed, estimates of the ionization fraction for different values of the temperature and the mass loss rate show that the gas is only partially ionized in the circumstellar envelope (e.g., Natta et al. 1988). Assuming $x_{\mathrm{e}}=0.4$ both the Brackett decrement and the ratio between the Paschen lines are well-reproduced, but the absolute fluxes are still too high by a factor 3-4. To obtain absolute fluxes in agreement with the observed ones, we have to assume low ionization fractions, down to $x_{\mathrm{e}}=0.15$. In this case, however, we are not able to reproduce the $\mathrm{Pa} \beta / \mathrm{Pa} \gamma$ ratio. We note that, in a non-spherical geometry, the lines become optically thick for lower mass accretion rates, and thus both a "thick" $\mathrm{Pa} / \mathrm{Pa} \gamma$ ratio and lower absolute fluxes can be obtained at the same time. In such a model, however, the profiles of the HI lines are predicted to be much narrower than the observed ones, i.e. FWHMs on the order of $100 \mathrm{~km} \mathrm{~s}^{-1}$ would be obtained, in contrast with our observations.

In conclusion, our analysis shows that neither a spherically symmetric wind nor spherically symmetric accretion can reproduce the Brackett and Paschen line ratios and fluxes. There are a few recent observational results suggesting that both the emission from the accretion columns and from a wind may contribute to the line profiles. Takami et al. (2001) and Whelan et al. (2004), for example, find that the gas from a wind contributes to the emission in the wings of their $\mathrm{H} \alpha$ and $\mathrm{Pa} \beta$ lines, thus producing broader line profiles than the ones predicted by magnetospheric accretion models. In our case, however, the observed $\mathrm{H}$ lines do not show the core/wing structure that is expected when both the accreting material and a wind are contributing to the emission.

To further investigate the possibility of a co-existence of emission from both inflowing and outflowing gas in our $\mathrm{H}$ lines we critically analyzed the spectro-astrometric results as described below.

\subsection{Insights from spectro-astrometry}

In the last two sections we have shown that both the accretion model and the wind model fail to reproduce all the characteristic of the observed NIR HI lines. A powerful technique for investigating the origin of the $\mathrm{HI}$ emission and separate the accretion and the wind contributions to the line profiles is spectroastrometry (see, e.g., Takami et al. 2001; Whelan et al. 2004, 2005). In fact, outflowing gas presents an extended emission testified by a detectable positional shift, while accreting gas remain concentrated on the source location.

Although Takami et al. (2001) detect extended emission in the wings of the $\mathrm{H} \alpha$ line up to $\sim 3 \mathrm{AU}$ from RU Lupi, we find that there is no spectro-astrometric signal in the HI NIR lines (see Fig. 4). The lack of signal indicates that either (i) the emission comes from a compact region (smaller than $\sim 1.3 \mathrm{AU}$ ) (ii) or, if there is a further emission from an extended region, this is symmetric, thus not detectable through spectro-astrometry, or too faint with respect to the emission from the star and the accretion columns (see below).

On the contrary, we do detect a substantial spectroastrometric signal in the HeI $\lambda 10830$ line after subtraction of possible biases (Fig. 4, panels $\mathrm{C}, \mathrm{C}^{\prime}$ ). The shift found in the HeI line is interpreted as an emission originating in the base of the jet, which partially fills the absorption feature of the "P Cygni" profile due to the inner wind. The inner stellar wind acts like a natural coronograph hiding the star along the blueshifted absorption, increasing the contrast of emission from outer regions. This could explain why we detect a positional shift in the HeI line and not in the NIR HI lines. 
A similar extended emission superimposed to the blueshifted absorption feature of the HeI line was found by Takami et al. (2002) in DG Tauri. Following the same reasoning as in Takami et al. (2002) we suggest that there are three contributions to the profile of the HeI line: (i) redshifted emission presumably due to accreting gas; (ii) blueshifted absorption due to the presence of an inner spherical wind emerging from the stellar surface; (iii) blue-shifted extended emission between $V \sim-50 \mathrm{~km} \mathrm{~s}^{-1}$ and $V \sim-200 \mathrm{~km} \mathrm{~s}^{-1}$ superimposed on the absorption feature and coming from a jet at a larger distance from the source. This emission is supposed to come from the base of the collimated microjet detected by Takami et al. (2001) in the forbidden [S II] and [O I] lines and in the $\mathrm{H} \alpha$ line. The velocity range covered by the $\mathrm{HeI}$ extended component is the same as in the $\mathrm{H} \alpha$ line. The HeI spectro-astrometric signal extends up to $\sim 20$ mas from the source ( $\sim 3 \mathrm{AU})$. However, as explained in Sect. 3.3, this is only a lower limit to the true angular scale of the emission region that could be more extended.

Combining in a bi-dimensional plane the shifts detected along the parallel and the perpendicular slits, we find that the extended emission is not completely aligned with the jet of Takami et al. (2001), the misalignment being not greater than $30^{\circ}$. This may be justified by the fact that (i) the jet may be not well-collimated in the He line; (ii) our observations were made 4 years later and a $200 \mathrm{~km} \mathrm{~s}^{-1}$ stream moves $1 \mathrm{AU}$ in a week. Furthermore, Takami et al. (2001) do observe variability in their spectro-astrometric data taken several years apart. Another discrepancy with respect to the result obtained by Takami et al. (2001) in the $\mathrm{H} \alpha$ line is that we did not detect extended emission in the redshifted part of the HeI line. This can be due, again, to the fact that the jet emission in the $\mathrm{HI}$ and $\mathrm{HeI}$ lines is fainter than in the optical $\mathrm{H} \alpha$ line and it can be shown by spectro-astrometry only over the blueshifted absorption, which hides the emission from the star and increases the contrast of outer emission.

\section{Conclusions}

In this paper we analyzed infrared spectra and images of the $\mathrm{T}$ Tauri star RU Lupi, in order to find observational constraints for the physics of the circumstellar region. This source has previously been observed in the optical wavelength range by other authors, the one reported about a strong accretion activity and the presence of a micro-jet detected in the optical forbidden lines and in the $\mathrm{H} \alpha$ line (Takami et al. 2001). We have observed RU Lupi in the NIR with high angular and spectral resolution for the first time. In this paper we report these observations and, in particular, we investigate the origin of the permitted $\mathrm{H}$ and He NIR lines through analysis of the line profiles and fluxes, and using the spectro-astrometry technique that allows us to constrain the emission region of each velocity component of the detected lines.

The problem of the origin of HI lines has been widely debated in the past decades. Many different models have been proposed to reproduce their profiles and fluxes: wind models (e.g., Hartmann et al. 1990), accretion models (e.g., Hartmann et al. 1994; Muzerolle et al. 1998a, 2001), and also hybrid models accounting for the emission from both the accretion columns and the wind (e.g., Kurosawa et al. 2006). These analyses, however, focused mainly on Balmer lines observed in the optical range. The Paschen and Brackett lines detected in our NIR spectra of RU Lupi present broad, slightly blueshifted, and nearly symmetric profiles, which are difficult to reproduce with either a wind or an accretion model. This has already been noted, in general, by Folha \& Emerson (2001), Whelan et al. (2004), and
Nisini et al. (2004). We tentatively used a toy model of a spherical envelope of partially ionized hydrogen with a wind or an accretion velocity profile (Nisini et al. 2004) to constrain the HI line ratios and fluxes. This analysis showed that neither a spherical wind nor spherical accretion can reproduce the Brackett and Paschen decrement and fluxes.

Our spectro-astrometric analysis did not highlight any extended emission in the Paschen and Brackett lines, suggesting that the region emitting the HI NIR lines is very compact $(<1.3 \mathrm{AU})$ and/or symmetric. On the other hand, the HeI $\lambda 10830$ line blueshifted absorption feature clearly indicates the presence of an inner wind. The wide range of velocities covered by the absorption feature favors the geometry of a spherical wind emerging from the stellar surface rather than a disk wind as found in many TTS by Edwards et al. (2006). In addition, the spectro-astrometric analysis highlighted the presence of an emission superimposed on the absorption feature that extends up to at least $\sim 3 \mathrm{AU}$ from the source and covers the same velocity range of the $\mathrm{H} \alpha$ extended emission found by Takami et al. (2001). We suggest that this emission comes from the blue lobe of the micro-jet detected in the optical lines by Takami et al. (2001).

Interestingly, the HeI showed itself to be more sensitive than the HI NIR lines to faint extended emission because the absorption feature increases the contrast between the emission from the extended region and the continuum from the source. This confirms the potential of the HeI $\lambda 10830$ line to investigate the complex geometry of the inner part of the circumstellar region of CTTS, where both the accretion and the ejection processes take place (see also Edwards et al. 2006). On the basis of these results, future high angular resolution observations of the permitted $\mathrm{H}$ and He lines in CTTS can give useful hints toward understanding the accretion/ejection mechanisms and the development of theoretical models accounting for the emission from both the outflowing and the accreting gas.

Acknowledgements. Linda Podio thanks the CAUP for hospitality during the development of this work. We are also grateful to the referee, Dr. Suzan Edwards, for the attentive comments that allowed us to improve the first version of this paper. This work was partially supported by the European Community's Marie Curie Research and Training Network JETSET (Jet Simulations, Experiments and Theory) under contract MRTN-CT-2004-005592, and by the Consiglio Nazionale delle Ricerche in the framework of a CNR/GRICES Agreement. P.J.V.G. work was supported in part by the Fundação para a Ciência e a Tecnologia through projects POCI/CTE-AST/55691/2004 and PTDC/CTEAST/65971/2006 from POCTI, with funds from the European program FEDER.

\section{References}

Alencar, S. H. P., Basri, G., Hartmann, L., \& Calvet, N. 2005, A\&A, 440, 595 Anderson, J. M., Li, Z.-Y., Krasnopolsky, R., \& Blandford, R. D. 2003, ApJ, 590, L107

Bacciotti, F., Mundt, R., Ray, T. P., et al. 2000, ApJ, 537, L49

Bailey, J. 1998, MNRAS, 301, 161

Beristain, G., Edwards, S., \& Kwan, J. 2001, ApJ, 551, 1037

Bouvier, J., Alencar, S. H. P., Harries, T. J., Johns-Krull, C. M., \& Romanova, M. M. 2007, Protostars and Planets V, 479

Brannigan, E., Takami, M., Chrysostomou, A., \& Bailey, J. 2006, MNRAS, 367, 315

Calvet, N., Hartmann, L., \& Hewett, R. 1992, ApJ, 386, 229

Castor, J. I. 1970, MNRAS, 149, 111

de Zeeuw, P. T., Hoogerwerf, R., de Bruijne, J. H. J., Brown, A. G. A., \& Blaauw, A. 1999, AJ, 117, 354

Dougados, C., Cabrit, S., Lavalley, C., \& Ménard, F. 2000, A\&A, 357, L61

Dupree, A. K., Brickhouse, N. S., Smith, G. H., \& Strader, J. 2005, ApJ, 625, L131

Edwards, S., Cabrit, S., Strom, S. E., et al. 1987, ApJ, 321, 473

Edwards, S., Fischer, W., Kwan, J., Hillenbrand, L., \& Dupree, A. K. 2003, ApJ, 599, L41 
Edwards, S., Fischer, W., Hillenbrand, L., \& Kwan, J. 2006, ApJ, 646, 319 Ferreira, J., Dougados, C., \& Cabrit, S. 2006, A\&A, 453, 785 Folha, D. F. M., \& Emerson, J. P. 2001, A\&A, 365, 90

Giovannelli, F., Vittone, A. A., Rossi, C., et al. 1995, A\&AS, 114, 341

Hartmann, L., Avrett, E. H., Loeser, R., \& Calvet, N. 1990, ApJ, 349, 168

Hartmann, L., Hewett, R., \& Calvet, N. 1994, ApJ, 426, 669

Herczeg, G. J., Walter, F. M., Linsky, J. L., et al. 2005, AJ, 129, 2777

Hummer, D. G., \& Storey, P. J. 1987, MNRAS, 224, 801

Kurosawa, R., Harries, T. J., \& Symington, N. H. 2006, MNRAS, 370, 580

Lamzin, S. A., Bisnovatyi-Kogan, G. S., Errico, L., et al. 1996, A\&A, 306, 877

Muzerolle, J., Calvet, N., \& Hartmann, L. 1998a, ApJ, 492, 743

Muzerolle, J., Hartmann, L., \& Calvet, N. 1998b, AJ, 116, 455

Muzerolle, J., Hartmann, L., \& Calvet, N. 1998c, AJ, 116, 2965

Muzerolle, J., Calvet, N., \& Hartmann, L. 2001, ApJ, 550, 944

Najita, J. R., Edwards, S., Basri, G., \& Carr, J. 2000, Protostars and Planets IV, 457
Natta, A., Giovanardi, C., \& Palla, F. 1988, ApJ, 332, 921

Natta, A., Testi, L., Comerón, F., et al. 2002, A\&A, 393, 597

Nisini, B., Milillo, A., Saraceno, P., \& Vitali, F. 1995, A\&A, 302, 169

Nisini, B., Antoniucci, S., \& Giannini, T. 2004, A\&A, 421, 187

Nisini, B., Bacciotti, F., Giannini, T., et al. 2005, A\&A, 441, 159

Podio, L., Bacciotti, F., Nisini, B., et al. 2006, A\&A, 456, 189

Pyo, T.-S., Hayashi, M., Kobayashi, N., et al. 2006, ApJ, 649, 836

Reipurth, B., Pedrosa, A., \& Lago, M. T. V. T. 1996, A\&AS, 120, 229

Rieke, G. H., \& Lebofsky, M. J. 1985, ApJ, 288, 618

Stempels, H. C., \& Piskunov, N. 2002, A\&A, 391, 595

Takami, M., Bailey, J., Gledhill, T. M., Chrysostomou, A., \& Hough, J. H. 2001, MNRAS, 323, 177

Takami, M., Chrysostomou, A., Bailey, J., et al. 2002, ApJ, 568, L53

Takami, M., Bailey, J., \& Chrysostomou, A. 2003, A\&A, 397, 675

Whelan, E. T., Ray, T. P., \& Davis, C. J. 2004, A\&A, 417, 247

Whelan, E. T., Ray, T. P., Bacciotti, F., et al. 2005, Nature, 435, 652 